\title{
Blastoschizomyces capitatus An Rare Opportunistic Infection Alerts the Clinical Correlation from a Territory care Hospital at Chennai
}

\author{
Dhevahi. E ${ }^{1}$, Sumathi. $S^{1}$ Bhavana Shree ${ }^{2}$, Sambantham $\mathrm{S}^{3}$ \\ Department of Microbiology ${ }^{l}, T B$ Chest Clinic ${ }^{2}$, Medical Superidendent ${ }^{3}$, Sri Muthukumaran Medical College \\ and Research Institute Chikarayapuram Chennai.
}

\begin{abstract}
Risk for new opportunistic infections has dramatically increased over the past decade because of the worldwide epidemic of HIV infection. Frequent and prolonged exposure of patients to a variety of environmental conditions has resulted in the recognition of infections with new fungal opportunists. Blastoschizomyces capitatus is less common emerging opportunistic fungal pathogen. Within a host, B.capitatus is able to grow in both unicellular yeast and multicellular filamentous forms. Here we describe two healthy cases attending TB chest clinic in our hospital with Blastoschizomyces capitatus infection and we review the clinical manifestations, microbiological characteristics which will help in elimination of the underlying predisposing factors which is crucial for clinical cure. Correct and timely identification of fungal specimens in clinical isolates is an essential component in the management of patients with fungal infections scientifically.
\end{abstract}

\section{Introduction:}

In recent decades, many fungal species have emerged as major causes of human disease.

In recent years, the frequency of opportunistic fungal infections has increased in parallel with the growing number of patients receiving aggressive chemotherapy. Blastoschizomyces capitatus, is a rare but wellestablished opportunistic infection. $(1,2)$ Blastoschizomyces capitatus has also been called Geotrichum capitatus and the teleomorph has been called Dipodascus capitatus. Blastoschizomyces capitatus may mostly be misdiagnosed morphologically as Trichosporon spp., or Candida krusei. While the lack of urease enzyme activity differentiates it from Trichosporon, its growth at $45^{\circ} \mathrm{C}$, resistance to cycloheximide and biochemical characteristics help in definitive identification (3-4).

We report two case of the emergence of disseminated infections caused by Blastoschizomyces capitatus at our TB chest clinic in our tertiary care Hospital near Mangadu, Central Chennai. Colonization of human mucosa may precede invasion and hematogenous dissemination, resulting in invasive tissue infection of mainly the lung, liver, and skin 5-7. Lack of preventative measures (vaccines) against all human fungal infections and the limited efficacy of increased resistance to the few $(<15)$ available antifungal drugs further complicate the issue, as risk-factors for opportunistic infections continue to increase in type, frequency and severity. Hence, it is important to correlate with clinical condition of the patient to know these diseases well so that we are able to manage them scientifically

Case 1

\section{Case Details :}

We report a first case of rare opportunistic fungus in a 52 year old man with a clinical diagnosis of Pulmonary Koch's with secondary fungal infection. Clinical history of productive cough since about 2 years, more since one month. Sputum was yellowish, mucoid, denies blood stained, not diurnal variation with shortness of breath-Grade2. Low grade fever off and on since about 2 months, evening rise associated with night sweats, loss of weight/ appetite, extreme fatigability.

Past history shows no prior H/O ATT or contact with PT Not a known hypertensive/ Diabetic/BA / COPD. On examination, patient was active, febrile, pallor+, anicteric, no clubbing/cyanosis/ pedal oedema, no significant palpable generalized lymphadenopathy. Chest X-Ray revealed extensive homogenous opacities on the left lung field.

Case 2

The second case is Male 68 years with a clinical diagnosis of COPD exacerbation with PT sequelae with a history of Productive cough since about 3 months, mucopurulent, whitish, non foul smelling, no risk suggesting hemoptysis. Insidious onset of shortness of breath grade II. No associated fever, loss of weight. Prior H/O ATT completed six month course about two years back. Current samples were negative for AFB Sputum was analyzed for bacteriological and fungal cultures. Chest X-Ray shows Fibrotic changes both apices. 
On admission: Both the patient was thoroughly evaluated \& investigated: Sputum was sent to the microbiology department for bacterial, fungal culture, Gram's and AFB stain. Laboratory reports show AFB positive. Culture was done on blood agar at $37^{\circ} \mathrm{C}$, Nutrient agar as a basal medium and Maconkey agar for bacterial culture, Sabouraud dextrose agar for fungal culture. Bacteriological reports show Gram's stain of Sputum with plenty of pus cells with Gram-positive cocci in short chains, pair's clusters. Fungal reports: Direct KOH examination showed fungal hyphae. Culture on SDA medium showed growth in the form of white to cream coloured colonies after five days mould-like, wrinkled, and develops short aerial hyphae with radiating edges by aging. On lactophenol cotton blue preparation and gram staining, true hyphae, pseudohyphae and annelloconidia resembling arthroconidia were seen microscopically. Further on subculture, growth was obtained at $45^{\circ} \mathrm{C}$ and on SDA in the presence of cyclohexamide. The isolated fungus was unable to utilize urea. Glucose and galactose were assimilated.

The texture of the colony is yeast-like, and white to cream colored. The isolate was identified as Blastoschizomyces capitatus. Further the cultures were sent to Department of Microbiology, Sri Ramachandra Medical College for confirmation. A repeat sample was also sent from both the cases to the laboratory for further confirmation of the organism.

On the basis of direct examination, culture and various biochemical tests, fungus was finally identified as Blastoschizomyces capitatus. Patient was put on Itraconazole $100 \mathrm{mg}$ od for five days. Patient responded well after seven days and was discharged after six days with instructions to continue treatment for the next 10 days. And a high protein diet advised with other supportive therapy with ATT through DOTS from nearest referral centre to residence as advice.

\section{Discussion}

Opportunistic fungal infections have emerged as important causes of morbidity and mortality in patients with severe underlying illnesses with compromised host defenses.10 Earlier Aspergillus spp.,Candida spp. collectively accounted for the majority of invasive and life-threatening fungal infections,11-13 but now as epidemiological trends indicate a shift towards infections by non-albicans Candida spp., as well as previously uncommon opportunistic fungi like Trichosporon beigelli, Fusarium and B. capitatus 14-16 Most fungal infections are caused by Candida spp., Aspergillus spp., but reports of infections caused by rare molds and unusual yeasts are increasing. 17,18.. The outcome of invasive disease caused by B. capitatus depends mainly on patients immunity. Several components of the case point to B. capitatus as being either the sole infectious agent causing disease or at least a significant contributor. Although B. capitatus is a colonizer of the normal respiratory tract, isolation of a pure culture of the organism suggests a role in the patient's clinical presentation.

Emerging fungal infections develop in a dynamic interplay of altered hosts, permissive environmental conditions, and selective antifungal pressures. Because of the lack of specific clinical, radiographic and histological features and the absence of diagnostic surrogate markers in blood, the diagnosis depends on the identification of the organism by means of culture based methods, where the conditions of PCR based study is not immediately feasible. Even more, the therapy of most emerging pathogens depends on the nature of fungal infection which leads to rapid progression with poor prognosis, as the patient was put on anti fungal, at the beginning to have a rapid recovery,

Finally, prompt diagnosis and aggressive treatment can only be achieved with increased awareness and better cooperation between clinicians, microbiologists and pathologists.

\section{Conclusion}

Rare emerging fungal infections are on the rise and fungal species that have been classically labeled as mildly-pathogenic or nonpathogenic are emerging as serious pathogens. Slow progress, in the development of more effective and safer antifungals drugs and the striking lack of fungal vaccines are not helping. This trend is strongly associated with improvements in disease management, diagnosis of infections and clinical course of manifestations. Therefore, use of definitive diagnostic procedures, rational application of available antifungals and prudent management of patients at risk are the more imperative.

With enlarging immunocompromised populations and the routine exposure of many of these patients to the environment outside the hospital, there is now greater potential for these low-virulence fungi to infect susceptible hosts. These emerging fungal pathogens present a challenge to the medical community, and it is essential that we provide basic training in medical mycology by means of epidemiologic reporting $[1,18]$. The apathy with regard to study of these opportunists is likely based on their relatively low frequency of infection and the select patient population that is affected.

Since our tertiary care centre shows a sudden rise on this rare and emerging fungal infection, this group of patients represent a major hurdle to their return to health. It becomes necessary that we continue to establish proper guidelines for diagnosis and management to improve diagnosis, prevention, and treatment of emergent 
mycoses, we must continue to educate the medical community at all levels of training and to investigate newer pathogenesis.

\section{Acknowledgments}

Author wishes to thank, Dr.Raveendran.T HOD Dr. Balamurugan.S TB Chest clinic of Sri Muthukumaran Hospital, Dr. Anupama HOD Mycology Department of Sri Ramachandra Medical College, and Dr.Jeevan, Dr.Nithyalakshmi, Dr.Sowmya, dr. Mohanakrishnan and the senior technicians -Arun Priya, Dharani and Karthik Dept. of Microbiology SMMCH \& RI for the kind support and help rendered for the isolating the organisms.

\section{References:}

[1]. Salkin I, Gordon M, Samsonoff W, Rieder C. Blastoschizomyces capitatus, a new combination. Mycotaxon 1985; 22:375- 380.

[2]. Wills T.S, Degrese A. et al. Blastoschizomyces capitatus pneumonia in an immunocompetant male. J South Med Assoc 2004; 97:702-704.

[3]. Collier, L., A. Balows, and M. Sussman. 1998. Topley \& Wilson's Microbiology and Microbial Infections, 9th ed, vol. 4. Arnold, London, Sydney, Auckland, New York.

[4]. Larone, D. H. 1995. Medically Important Fungi - A Guide to Identification, 3rd ed. ASM Press, Washington, D.C.

[5]. Girmenia C, Pagano L, Martino B, D’Antonio D, Fanci R, Specchia G, et al. Invasive infections caused by Trichosporon species and Geotrichum capitatum in patients with hematological malignancies: a retrospective multicenter study from Italy and review of the literature. J Clin Microbiol. 2005;43:1818-28. doi:10.1128/ JCM.43.4.1818-1828.2005

[6]. Martino R, Salavert M, Parody R, Tomas JF, de la Camara R, Vazquez L, et al. bastoschizomyces capitatus infection in patients with leukemia: report of 26 cases. Clin Infect Dis. 2004;38:335-41.

[7]. Martino P, Venditti M, Micozzi A, Morace G, Polonelli L, Mantovani MP, et al. blastoschizomyces capitatus: an emerging cause of invasive fungal disease in leukemia patients. Rev Infect Dis. 1990;12:570-82. doi:10.1093/clinids/12.4.570

[8]. Martino R, Salavert M, Parody R, Tomas JF, de la Camara R, Vazquez L, et al. bastoschizomyces capitatus infection in patients with leukemia: report of 26 cases. Clin Infect Dis. 2004;38:335-41. doi:10.1086/380643

[9]. Martino P, Venditti M, Micozzi A, Morace G, Polonelli L, Mantovani MP, et al. blastoschizomyces capitatus: an emerging cause of invasive fungal disease in leukemia patients. Rev Infect Dis. 1990;12:570-82. doi:10.1093/clinids/12.4.570

[10]. Duong TA. Infection due to Penicillium marnefei, an emerging pathogen: review of 155 reported cases. Clin Infect Dis 1996;23:125130.

[11]. Groll AH, Shah PM, Mentzel C, Schneider M, Just-Nuebling G, Huebner K. Trends in the postmortem epidemiology of invasive fungal infections at a university hospital. J Infection 1996; 33:23-32.

[12]. Nguyen MH, Peacock JE Jr, Moms AJ et al. The changing face of candidemia: emergence f non-Candida albicans species and antifungal resistance. Am J Med 1996;100:617- 623.

[13]. Wald A, Leisenring W, Van Bunk JA, Bowden RA. Epidemiology of Aspergillus infections in a large cohort of patients undergoing bone marrow transplantation. J Infectious Dis 1997;175:1459-1466.

[14]. Vamvarian SE, Anaissie EJ, Bodey GP. Emerging fungal pathogens in immunocompromised patients: classification, diagnosis, and management. Clin Infect Dis 1993; 17 (Suppl. 2): S487-491.

[15]. Cunliffe NA, Denning DW. Uncommon invasive mycoses in AIDS. 1995;9:411-420.

[16]. Perfect JR, Schell WA. The new fungal opportunists are coming. Clin Infect Dis 1996; 22 (Suppl. 2): S112-118.

[17]. Malani AN, Kauffman CA. Changing epidemiology of rare mould infections: implications for therapy. Drugs. 2007;67:1803-12.

[18]. Imhof A, Balajee SA, Fredricks DN, Englund JA, Marr KA. Breakthrough fungal infections in stem cell transplant recipients receiving voriconazole. Clin Infect Dis. 2004;39:743-6.

Figure 1. Sabouraud's Dextrose Agar with cycloheximide and Sabouraud's Dextrose Agar incubated at $45^{\circ} \mathrm{C}$ showing cream coloured yeast like colonies of Blastoschizomyces capitatus

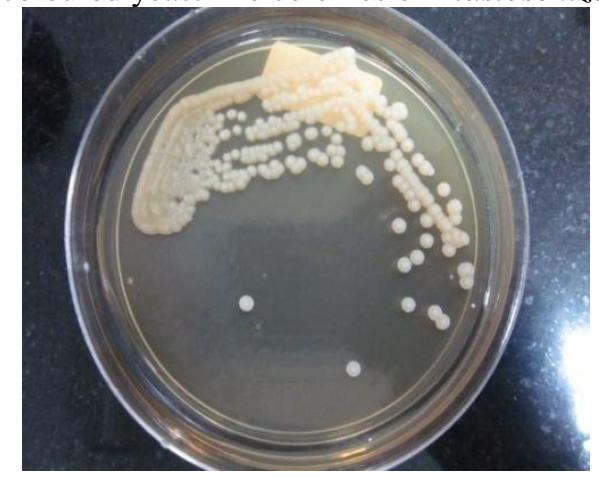


Figure 2. Lactophenol Cotton blue mount showing annelloconidia and pseudohyphae

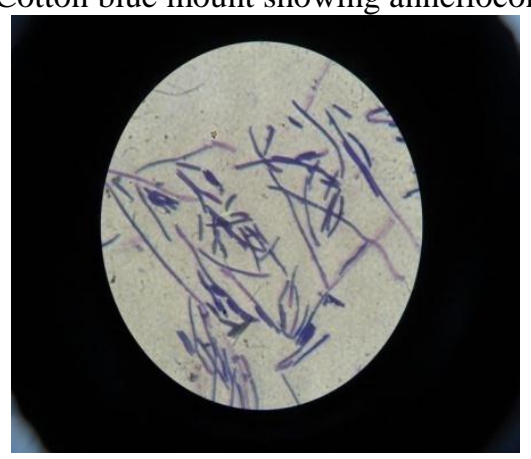

